\title{
AVALIAÇÃO DAS CARACTERÍSTICAS MECÂNICAS DO PLA, IMPRESSA EM 3D, PARA APLICAÇÃO EM PRÓTESES EM ANIMAIS DE PEQUENO E MÉDIO PORTE
}

Andressa Pereira dos Reis Croucillo ' José Dimas de Arruda ' Luciano José Vieira Franco ' Jussara Fernandes Leite ' Mayara Rezende Carvalho ${ }^{2}$

\section{Resumo}

O desenvolvimento de impressoras 3D permitiu uma maior produção de peças e próteses médicas provenientes de polímeros termoplásticos como PLA, PETG e ABS. Este trabalho avaliou propriedades mecânicas do PLA, impressas na forma de filamento, em determinada temperatura, para aplicação de próteses mecânicas externas para animais de pequeno e médio porte. Foram impressos diversos corpos de provas, para a realização de testes de tração, verificando que a configuração com densidade $100 \%$, espessura de $0,4 \mathrm{~mm}$ e direção de impressão de $45^{\circ}$ e $135^{\circ}$ apresentou o melhor resultado de resistência mecânica. Realizaram-se também, simulações matemáticas para análise de resistência à queda $\mathrm{e}$ flambagem. O conjunto de testes físicos e matemáticos determinou que o PLA não apresenta resistência mecânica e ao impacto suficiente para a produção de próteses.

Palavras-chave: PLA; Polímero; Impressora 3D; Próteses.

\section{EVALUATION OF THE MECHANICAL CHARACTERISTICS OF A PROSTATE FOR SMALL AND MEDIUM PUPPIES OF PLA MATERIAL IMPRESSED IN 3D}

\begin{abstract}
The development of 3D printers have allowed a greater production of medical parts and prosthesis from thermoplastic polymers such as PLA and ABS. This work has evaluated the mechanical characteristics of PLA, printed in the form of filaments, at a certain temperature, for application of external mechanical prosthesis for small and medium animals. Several specimens were used to perform tensile tests, verifying that the configuration with a density of $100 \%$, a thickness of $0.4 \mathrm{~mm}$ and a printing direction of $45^{\circ}$ and $135^{\circ}$ showed the best mechanical resistance. Also, mathematical simulations were performed for the analysis of resistance to fall and buckling. The set of physical and mathematical tests determined that the PLA does not have enough mechanical resistance to impact for the production of prostheses.
\end{abstract}

Keywords: PLA; Polymer; Printer 3D; Prostheses.

\section{INTRODUÇÃO}

Segundo Alves [I], o desenvolvimento de produtos para saúde, de pessoas ou animais com necessidades específicas, estão cada vez mais diversificadas e exigentes quanto à necessidade de personalização. Denominados Tecnologia Assistiva (TA), constituem-se em dispositivos desenvolvidos para permitir uma maior funcionalidade aos envolvidos, com incapacidades ou mobilidade reduzida, no auxílio de suas atividades da vida diária. Dentre estes, se enquadram órteses ou próteses, dispositivos mecânicos extracorpóreos com o objetivo de aumentar a independência, funcionalidade e desempenho biomecânico dos utilizadores.

Selhorst [2] na busca pela utilização de novas tecnologias em vários setores como os da indústria e do comércio de produtos para saúde, afirma que a prototipagem

'Fundação Presidente Antônio Carlos, Conselheiro Lafaiete, MG, Brasil. E-mail: jdimasarrudaem@yahoo.com.br ${ }^{2}$ Instituto de Educação Superior Vale do Ipiranga, Conselheiro Lafaiete, MG, Brasil.

*Versão revisada de trabalho apresentado na ABM Week 20I7, de 2 a 6 de outubro de 20 I7, São Paulo, SP, Brasil. 
rápida se apresenta como uma solução de grande interesse, quando se leva em consideração o custo e o tempo de incorporação da mesma. Afirma que comparado aos processos tradicionais, como a usinagem, a impressão 3D atende com maior rapidez, mas essas podem trabalhar de modo cooperativo, uma suprindo as necessidades da outra, uma vez que são processos que necessitam de várias linhas para acabamento. Para Todd [3], uma das principais aplicações da prototipagem rápida é na visualização antecipada de novos projetos, utilizando modelagens computacionais, quando estes ainda estão em fase de avaliação ou mesmo em fase de concepção mais avançada.

Celentano [4] afirma que a melhoria da tecnologia e sofisticação de filamentos empregada nas construções de novas e modernas impressoras, têm-se tornado cada vez maior. Atualmente, podem-se encontrar polímeros, compósitos, alimentos e até mesmo tecidos humanos sendo utilizados para impressão dos mais diversos produtos.

A produção de próteses mecânicas para animais, utilizando a prototipagem rápida, é realizada comumente com dois materiais poliméricos, o Acrilonitrila butadieno estireno - ABS e o Politereftalato de etileno glicol - PETG. Independente do material aplicado, Silva [5] afirma que a resistência mecânica é maior quanto maior for a densidade do mesmo. O ABS é um termoplástico leve e rígido, possui boa flexibilidade e resistência ao impacto, podendo ter diversas formas e cores, através da moldagem térmica a altas temperaturas e adições de pigmentos. Suas características permitem uma boa aplicação deste na utilização de próteses mecânicas, afirma Celentano [4]. Sanches [6] realizou ensaios de tração no material $A B S$ normal e envelhecido, observando a ruptura deste em valores de tensões superiores a $25 \mathrm{MPa}$, o que qualifica o material para tal aplicação.

Reis [7] afirma que o PETG, termoplástico derivado do petróleo, reciclável como o PET, é utilizado na indústria há vários anos, recentemente impresso em 3D, com aplicações em tubulações, próteses e brinquedos. Resistente a altas temperaturas, porém inferior ao ABS, é aplicável a peças que precisem de transparência ou encaixes com maior flexibilidade e alta resistência mecânica. Um grande diferencial desse material é sua baixa reatividade a diversos tipos de solventes do mercado. Não possui contra indicações de uso. Quanto às características ecológicas, PETG é melhor que o $A B S$, por ser reciclável.

De acordo com Simões et al. [8], o PLA - Poli (ácido lático), ecologicamente, é um termoplástico biodegradável (Bioplástico) derivado de fontes renováveis como amido de milho, e cana de açúcar. É um material rígido e resistente, difícil de deformar ou flexionar, com uma alta dureza, que o torna com baixa resistência ao impacto. Sua alta fluidez e baixa contração durante o processo de extrusão permite a produção de peças com alta precisão dimensional e bom acabamento superficial. É um material indicado para produção de protótipos que não sejam submetidos às condições de altos esforços mecânicos, atritos ou altas temperaturas. Estes fatores implicam no processo de desgaste deste material. Possui ponto de fusão entre $190-220{ }^{\circ} \mathrm{C}$, alongamento máximo de $4,43 \%$, resistência e módulo de flexão $65 \mathrm{MPa}$ e 2,5GPa, respectivamente, além da tensão de rendimento no valor de $62,63 \mathrm{MPa}$.

\section{MATERIAIS E MÉTODOS}

É apresentada uma metodologia para análise de resistência mecânica de material polimérico para construção de próteses externas de animais de pequeno porte e médio. Foram utilizados Poli (ácido lático) - PLA na construção de corpos de prova para ensaio de tração de acordo com a norma ASTM D638, Figura Ia. Esses corpos de provas foram obtidos por impressão 3D, utilizando uma impressora SETHI AIP 3, Figura Ib, com temperatura de mesa em

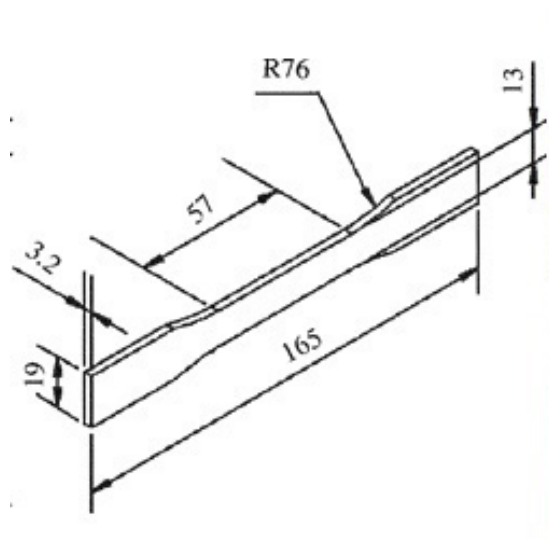

a)

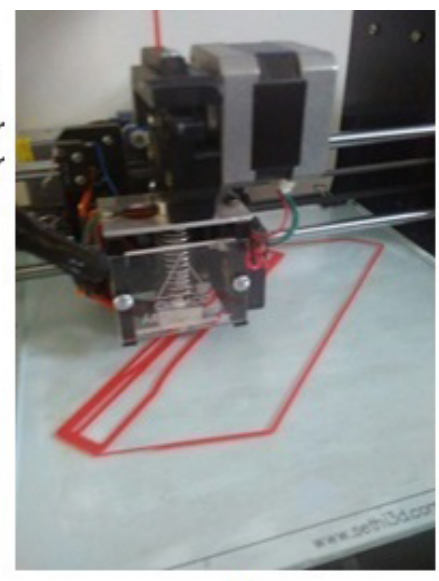

b)

\begin{tabular}{|c|c|c|}
\hline DENSIDADE & $\begin{array}{c}\text { ESPESSURA } \\
\text { DE } \\
\text { CAMADA } \\
\text { (mm) }\end{array}$ & $\begin{array}{c}\text { DIREÇÃo } \\
\text { DE } \\
\text { IMPRESSÃo }\end{array}$ \\
\hline $20 \%$ & 0,2 & $0^{\circ} / 45^{\circ}$ \\
\hline $20 \%$ & 0,2 & $90^{\circ} / 135^{\circ}$ \\
\hline $20 \%$ & 0,4 & $0^{\circ} / 45^{\circ}$ \\
$20 \%$ & 0,4 & $90^{\circ} / 135^{\circ}$ \\
\hline $100 \%$ & 0,2 & $0^{\circ} / 45^{\circ}$ \\
$100 \%$ & 0,2 & $90^{\circ} / 135^{\circ}$ \\
\hline $100 \%$ & 0,4 & $0^{\circ} / 45^{\circ}$ \\
\hline $100 \%$ & 0,4 & $90^{\circ} / 135^{\circ}$ \\
\hline
\end{tabular}

c)

Figura I. (a) Modelo de corpo de prova ASTM D638 (dimensões em milímetros); (b) Impressora SETHI AIP 3; (c) Características dos corpos de provas impressos. (Pesquisa Direta, 2016). 
$60{ }^{\circ} \mathrm{C}$, temperatura de extrusor em $200{ }^{\circ} \mathrm{C}$ e filamento de espessura $1,5 \mathrm{~mm}$. As variáveis foram densidade de impressão, direção e espessura da camada de impressão, como mostrado na Figura Ic. Os ensaios foram feitos em triplicata, e os resultados plotados a partir do Matlab.

Realizaram-se ensaios mecânicos de tração, com o objetivo de analisar a resistência mecânica do material a ser empregado na construção das próteses. A máquina de tração utilizada foi a DL5000/10000, com capacidade de carga de até $10.000 \mathrm{kgf}$, que compõe o conjunto instalado no Laboratório de Caracterização de Materiais da Fundação Presidente Antônio Carlos.

Além desses, foram realizadas simulações matemáticas no Solidworks, avaliando a resistência quanto à flambagem e queda, em um modelo de prótese com altura de $15 \mathrm{~cm}$ e raio de $4 \mathrm{~cm}$. Foram utilizados corpo sólido de PLA, modelo isotrópico linear elástico, com resistência a tração teórica de $62,63 \mathrm{~N} / \mathrm{mm}^{2}$, densidade $800 \mathrm{~kg} / \mathrm{m}^{3}$, módulo de elasticidade $2000 \mathrm{~N} / \mathrm{mm}^{2}$ e coeficiente de poison 0,43 . Utilizou-se para fins de cálculos, um modelo de animal com peso entre $10 \mathrm{~kg}$ e $20 \mathrm{~kg}$, para avaliar as características mecânicas do material na aplicação desejada.

\section{RESULTADOS E DISCUSSÃO}

\section{I Ensaios de Tração}

A Figura 2a mostra a curva tensão $\times$ deformação para o corpo de prova com densidade de $20 \%$, direção de impressão da camada $0^{\circ} / 90^{\circ}$, e espessura de $0,2 \mathrm{~mm}$. Esta configuração do material suportou uma tensão de $25,55 \mathrm{KPa}$, atingindo uma deformação máxima de 0,07 . A Figura $2 b$ é uma análise semelhante com o corpo de prova variando apenas a espessura para $0,4 \mathrm{~mm}$. Observa-se uma tensão máxima de 20,53KPa com deformação de 0,05I. Além de suportar maiores tensões, o material com espessura de $0,2 \mathrm{~mm}$ apresentou um início de deformação plástica, comportamento não observado para a espessura de $0,4 \mathrm{~mm}$. Os dois corpos de provas fraturaram com um tempo inferior a I0s de teste.

Para as análises da Figura 3a e 3b, a direção da impressão da camada do PLA foi $45^{\circ} / 135^{\circ}$ e densidade de $20 \%$. Obteve-se uma tensão de $25,86 \mathrm{KPa}$ e deformação máxima de 0,068 para a espessura de $0,2 \mathrm{~mm}$. Para a espessura de $0,4 \mathrm{~mm}$ estes valores foram $22,67 \mathrm{KPa}$ e 0,086 respectivamente. Novamente, o material que suportou maior tensão foi o de menor espessura de filamento, porém, apresentando menor deformação plástica. Este corpo de prova com $0,4 \mathrm{~mm}$ de espessura do filamento fraturou com um tempo de teste $20 \%$ maior que o de espessura $0,2 \mathrm{~mm}$.

Comparando todas as configurações com densidade $20 \%$, observou-se que para a espessura $0,2 \mathrm{~mm}$, Figuras $2 \mathrm{a} \mathrm{e} 3 \mathrm{a}$, o comportamento das curvas são semelhantes, com valores de tensão e deformação próximos. Para a espessura de $0,4 \mathrm{~mm}$, Figuras $2 \mathrm{~b}$ e $3 \mathrm{~b}$, ambas as direções de impressão suportaram tensões aproximadas, porém o material com direção de impressão de $45^{\circ} / 135^{\circ}$ apresentou maior deformação plástica. Dentre as quatro configurações para a densidade de $20 \%$, a direção de $45^{\circ} / 135^{\circ}$ e espessura $0,4 \mathrm{~mm}$ foi a que apresentou maior ductilidade.
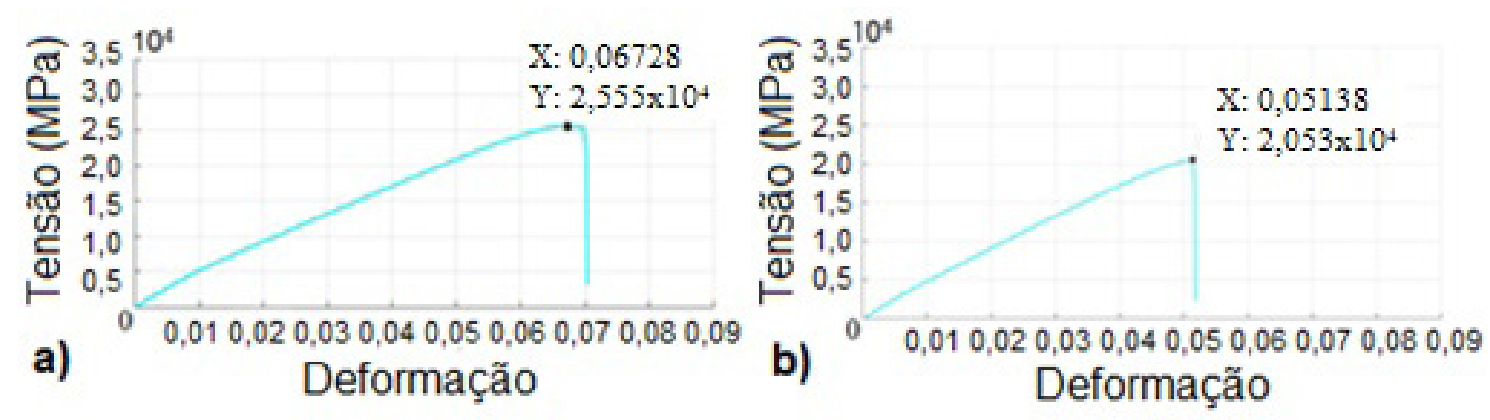

Figura 2. Gráfico tensão $\times$ deformação PLA: $\rho=20 \%$ e direção $=0^{\circ} / 90^{\circ}$. (a) espessura $=0,2 \mathrm{~mm}$; (b) espessura $=0,4 \mathrm{~mm}$.
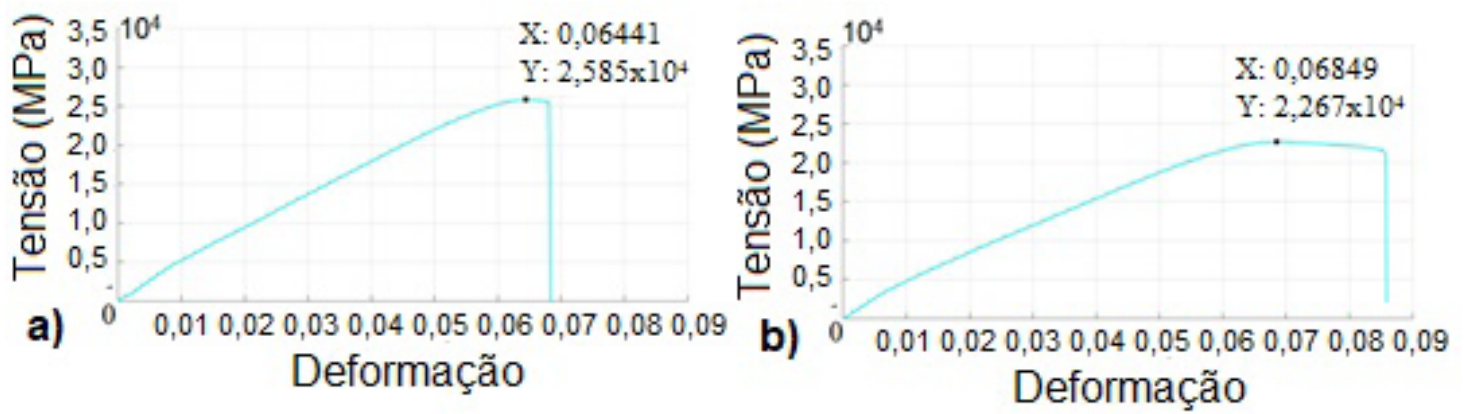

Figura 3. Gráfico tensão $\times$ deformação PLA: $\rho=20 \%$ e direção $=45^{\circ} / 135^{\circ}$. (a) espessura $=0,2 \mathrm{~mm}$; (b) espessura $=0,4 \mathrm{~mm}$. 
Analisou-se também o material impresso com densidade de $100 \%$. As Figuras 4a e 4 b mostram a configuração para direção de impressão de $0^{\circ} / 90^{\circ}$, com espessuras de $0,2 \mathrm{~mm}$ e 0,4 mm, respectivamente. $\mathrm{O}$ material de menor espessura suportou uma tensão máxima de 33,85KPa e uma deformação total de 0,073 , enquanto o corpo de prova impresso com 0,4 mm de espessura suportou uma tensão máxima de 34,27KPa e uma deformação total de 0,062. A carga máxima suportada pelo PLA não variou quanto à espessura de impressão, mas uma maior deformação total foi observada para a espessura de impressão igual a $0,2 \mathrm{~mm}$.

Os resultados para o material com densidade de $100 \%$ e direção de impressão de $45^{\circ} / 135^{\circ}$, são apresentados na Figura 5a, com espessura 0,2 mm, observando uma tensão máxima de 34,8KPa e deformação de 0,065; a Figura 5b, para a espessura $0,4 \mathrm{~mm}$, apresenta uma tensão máxima de $24,40 \mathrm{KPa}$ e uma deformação de 0,088 . O material com espessura de 0,4 mm atingiu um maior valor de deformação, suportando um tempo $25 \%$ maior de processo se comparado à espessura de $0,2 \mathrm{~mm}$, para a densidade $100 \%$ e direção de impressão $45^{\circ} / 35^{\circ}$.

Comparando os materiais de densidade $100 \%$ e espessura $0,2 \mathrm{~mm}$, variando a direção de camada impressa $0^{\circ} / 90^{\circ}$, Figura $4 a$, e $45^{\circ} / 135^{\circ}$, Figura $5 a$, observa-se que os dois materiais rompem em tensões aproximadamente iguais, porém a direção $0^{\circ} / 90^{\circ}$ apresenta uma deformação plástica superior. Fazendo a mesma comparação para a espessura de $0,4 \mathrm{~mm}$, direção de camada impressa $0^{\circ} / 90^{\circ}$, Figura $4 \mathrm{~b}$, suportou uma maior tensão aplicada, mas a impressão na direção $45^{\circ} / 135^{\circ}$, Figura $5 \mathrm{~b}$, apresentou uma maior deformação plástica.

Comparando as duas densidades estudadas observou-se que o material suportou maiores tensões até a ruptura para densidade de 100\%, mostrando a importância da compactação estrutural (densidade) do material durante sua produção, de acordo com Silva [5]. Observou-se também que os maiores valores de deformação foram observados na direção de impressão $45^{\circ} / 135^{\circ}$ e densidade de $100 \%$. Esta análise implica que o PLA possui maior resistência mecânica em maiores densidades de impressão, na direção a $45^{\circ}$ em relação à direção normal das cadeias carbônicas.

A variação de resistência mecânica encontrada nas configurações estudadas foi pequena, e nenhuma das situações determinou a possibilidade de empregar este material com segurança em próteses de animais, como proposto inicialmente. A resistência mecânica encontrada para o PLA está coerente com os dados de Simões et al. [8], baixa ductilidade, o que leva a fragilidade do material. A não garantia de aplicação do PLA em substituição ao $A B S$ não descarta completamente a aplicação deste em próteses que não exija grandes esforços mecânicos.

\subsection{Simulações Computacionais do PLA}

Realizaram-se simulações computacionais para o teste de queda, que mostra o perfil de tensões ao longo de um corpo de prova de PLA, Figura 6a, além do teste de flambagem, mostrado na Figura 6b.

No teste de queda, variaram-se as tensões para calcular as possíveis deformações em um impacto inicial do produto com uma superfície rígida ou flexível. A simulação foi feita com uma carga de $20 \mathrm{~kg}$. À medida que o corpo de prova se deformava, os impactos internos e externos secundários eram calculados, localizando fragilidades ou pontos de falha, assim como tensões e deslocamentos. Observaram-se concentrações de tensão na superfície do corpo de prova,
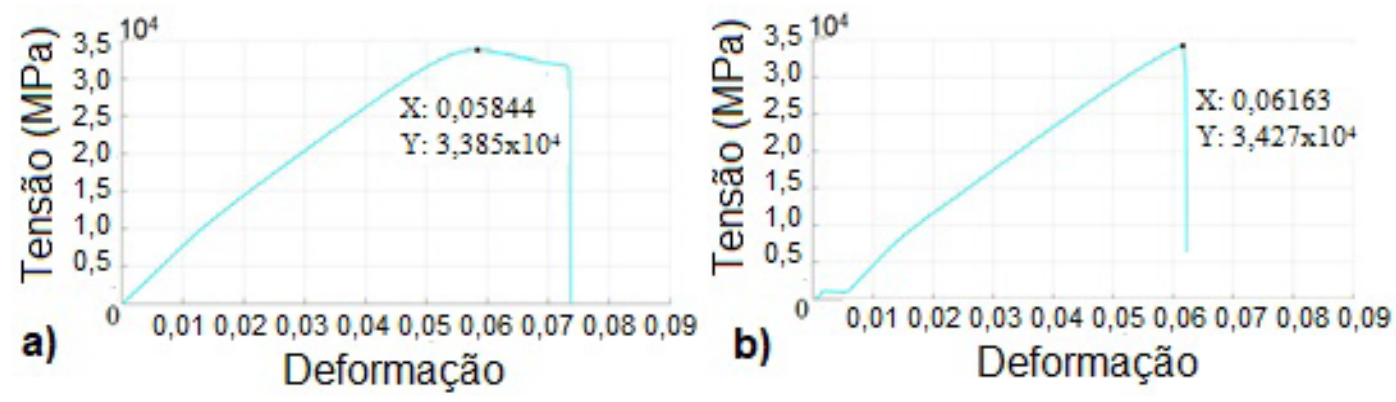

Figura 4. Gráfico $\times$ tensão deformação PLA: $\rho=100 \%$; Direção $=0 \% 90^{\circ}$. (a) espessura $=0,2 \mathrm{~mm}$; (b) espessura $=0,4 \mathrm{~mm}$.
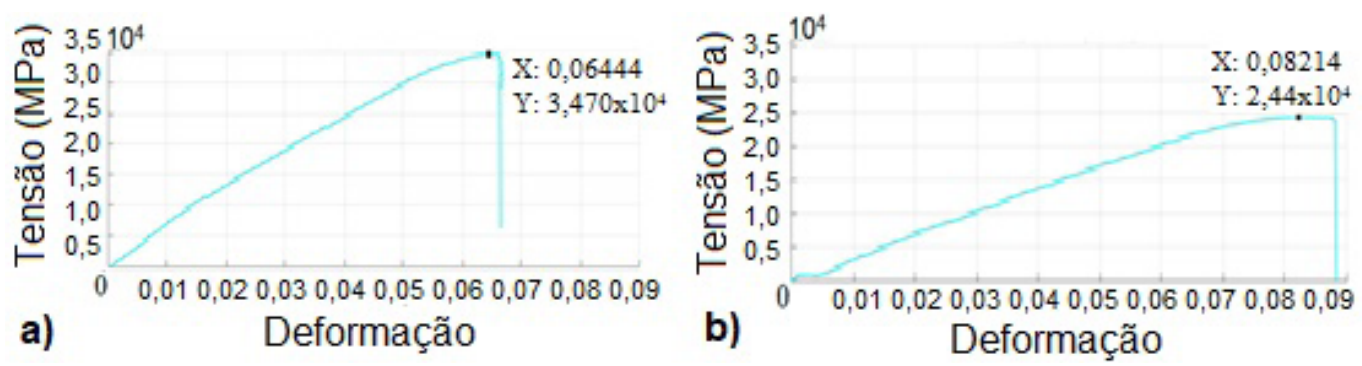

Figura 5. Gráfico tensão $\times$ deformação PLA: $\rho=100 \%$; Direção $=45^{\circ} / 135^{\circ}$. (a) espessura $=0,2 \mathrm{~mm}$; (b) espessura $=0,4 \mathrm{~mm}$. 


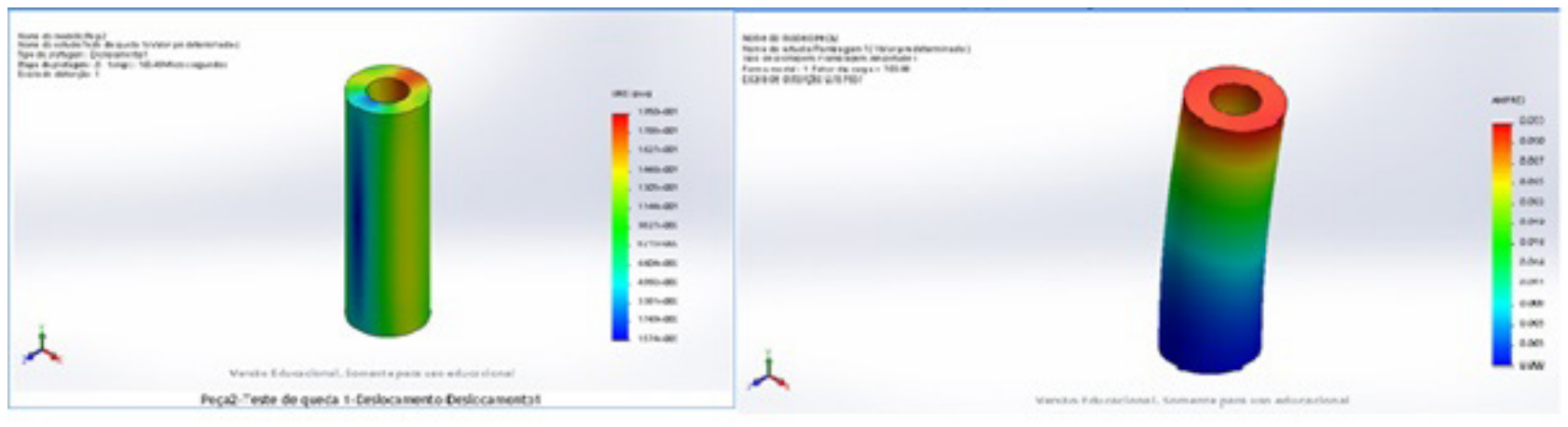

Figura 6. (a) Simulação do teste de queda; (b) Simulação do teste de flambagem.

local que sofreria maior desgaste devido à carga aplicada. O teste de queda apresentou um bom aspecto, mas com características de material frágil, com ruptura eminente devido ao impacto, como apresentado por Simões et al. [8]. Na simulação de flambagem, uma extremidade foi fixada, e na outra foi aplicado a carga. $O$ fenômeno de flambagem foi observado quando se aplica uma força de $20 \mathrm{~kg}$, diminuindo a vida útil da prótese.

\section{CONCLUSÕES}

Os resultados permitem inferir que:

- Os ensaios mecânicos realizados nos corpos de prova de PLA mostraram uma baixa resistência mecânica para que este seja aplicado em próteses de animais que exijam resistência ao impacto devido à sua baixa ductilidade;

- O método de impressão que competiu ao material uma maior resistência mecânica foi o de densidade $100 \%$. A direção de impressão $45^{\circ} / 135^{\circ}$ apresentou melhores valores de ductilidade, apesar de todas direções apresentarem baixos valores de deformação;

- Nas simulações matemáticas do Solidwoks foi verificado concentrações de tensões na superfície do corpo de prova além do fenômeno de flambagem. Estes resultados dificultam a aplicação deste material na construção de próteses, reduzindo o campo de aplicação destas em próteses que não exijam grandes esforços mecânicos.

\section{REFERÊNCIAS}

I Alves DCC. Aspectos ergonómicos relevantes para a concepção de tecnologia assistiva: órteses de membros inferiores. Braga: Universidade do Minho; 2012.

2 Selhorst A Jr. Análise comparativa entre os processos de prototipagem rápida na concepção de novos produtos: um estudo de caso para determinação de processo mais indicado. Curitiba: Pontifícia Universidade Católica do Paraná; 2008.

3 Todd G. User's guide to rapid prototyping. Dearborn: Society of Manufacturing Engineers; 2004.

4 Celentano CGJTD. Caracterización mecánica de materiales termoplásticos de uso en componentes de refrigeradores. In: Anais do $3^{\circ}$ Congreso Internacional en Ciencia y Tecnología de Metalurgia y Materiales; 2003; Argentina. Argentina: CONAMET; 2003. p. 298-30I. (no. 4).

5 Silva KIM. Preparação de nanocompósitos de PLA/PEAD/TiO2 degradáveis [monografia]. Porto Alegre: UFRGS; 2014.

6 Sanches EMS, Felisberti MI. Propriedades mecânicas de tração do ABS pós consumo. In: Anais do $6^{\circ}$ Congresso Brasileiro de Polímeros; 200 I; Gramado. Gramado: CBPol; 200 I. p. I099-I I 0 I.

7 Reis T. Conheça os diferentes tipos de materiais para impressão 3D FDM. Cascavel: Impressão 3D Fácil; 2016 [acesso em 5 out. 2016]. Disponível em: http://www.impressao3dfacil.com.br/conheca-os-diferentes-tipos-demateriais-para-impressao-3d-fdm/

8 Simões CL, Viana JC, Cunha AM. Mechanical properties of poly(lactic acid) blends. Journal of Applied Polymer Science. 2009; I I 2( I):345-352.

Recebido em: 20 Nov. 2017

Aceito em: 14 Ago. 2018 